Document downloaded from:

http://hdl.handle.net/10251/74488

This paper must be cited as:

Zomeño Segado, C.; Juste Aguilera, V.; Hernández, P. (2012). Application of NIRS for predicting fatty acids in intramuscular fat of rabbit. Meat Science. 91(2):155-159. doi:10.1016/j.meatsci.2012.01.009.

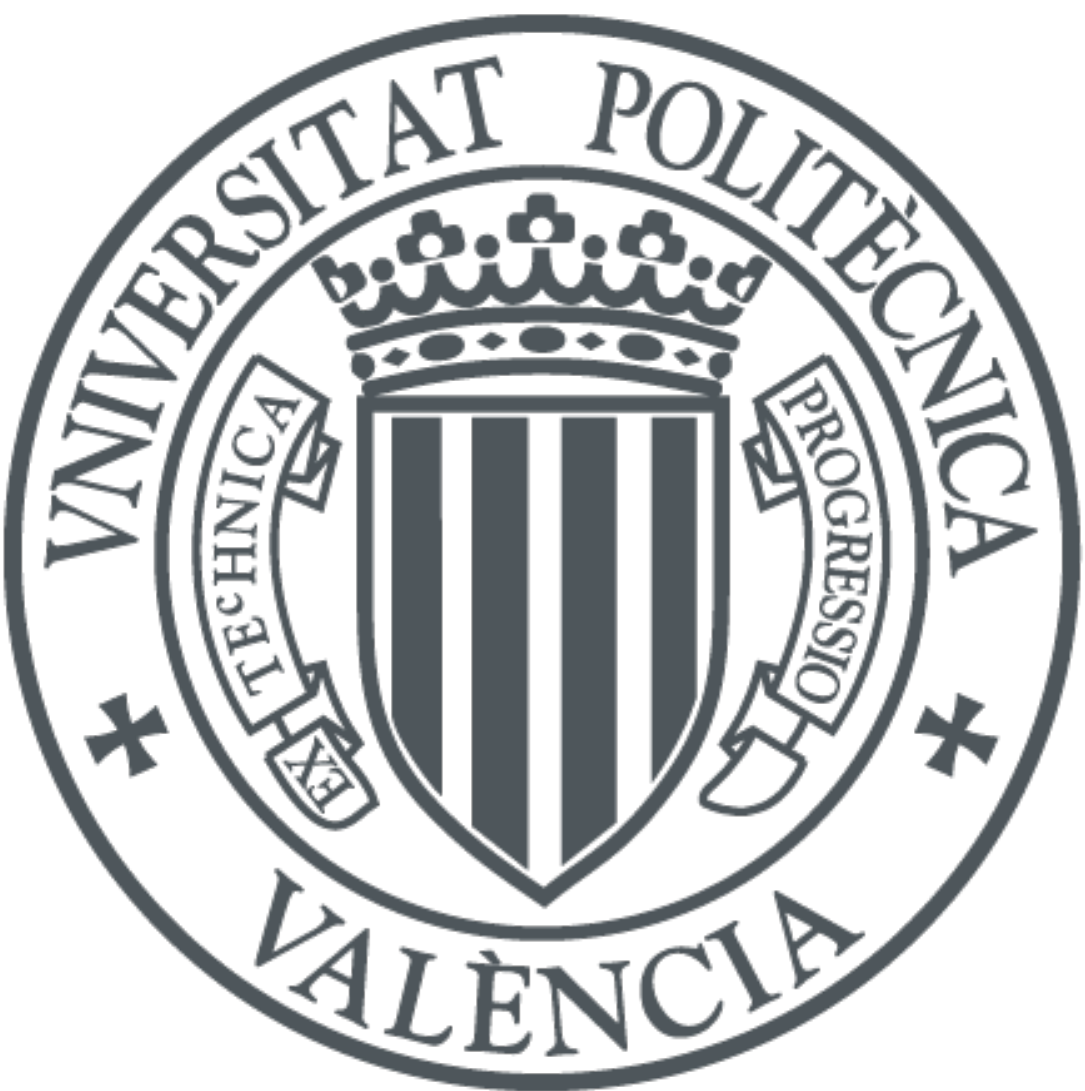

The final publication is available at

https://dx.doi.org/10.1016/j.meatsci.2012.01.009

Copyright Elsevier

Additional Information 


\section{Application of NIRS for predicting fatty acids in intramuscular fat of rabbit}

Zomeño, C., Juste, V., Hernández, P. 

ABSTRACT: The aim of this study was to evaluate the use of near infrared reflectance spectroscopy (NIRS) for predicting fatty acid content in intramuscular fat (IMF) to be applied in rabbit selection programmes. One hundred and forty three freeze-dried Longissimus muscles (LM) were scanned by NIRS (1100-2498 nm). Modified Partial Least Squares models were obtained. Equations were selected according to standard error of cross validation (SECV) and coefficient of determination of cross validation $\left(R^{2} \mathrm{CV}\right)$. Residual predictive deviation of cross validation (RPD $\mathrm{CV}$ ) was also studied. Accurate predictions were reported for IMF $\left(R^{2} c V=0.98 ; R P D_{C V}=7.57\right)$, saturated $\left(R^{2} \mathrm{CV}=0.96 ; \quad R P D_{C V}=5.08\right)$ and monounsaturated fatty acid content $\left(R^{2} c V=0.98\right.$; $\left.R P D_{C V}=6.68\right)$. Lower accuracy was obtained for polyunsaturated fatty acid content $\left(R^{2} c V=0.83 ; R P D_{C V}=2.40\right)$. Several individual fatty acids were accurately predicted such as C14:0, C15:0, C16:0, C16:1, C17:0, C18:0, C18:1 n-9, C18:2 n-6 and C18:3 n-3 $\left(R^{2} \mathrm{cV}=0.91-0.97 ; R P D_{c v}>3\right)$. Long chain polyunsaturated fatty acids and $\mathrm{C} 18: 1 \mathrm{n}-7$ presented less accurate prediction equations $\left(R^{2} c V=0.12-0.82 ; R P D_{c V}<3\right)$.

Keywords: fatty acids, intramuscular fat, NIRS, rabbit.

\section{INTRODUCTION}

Rabbit meat offers good nutritive and dietetic properties because it has lower fat and higher polyunsaturated fatty acid (PUFA) content than other meats (Hernández and Gondret, 2006). The most ubiquitous fatty acids (FA) are palmitic (C16:0), oleic (C18:1 $n-9)$ and linoleic (C18:2 n-6) acids, showing percentages higher than $20 \%$ of total FA. Rabbit meat also contains high protein content and high levels of essential amino acids compared to other meats (Hernández and Dalle Zote, 2010).

Conventional methods used to determine meat chemical composition are laborious, expensive, time-consuming and destructive. In contrast, near infrared reflectance spectroscopy (NIRS) is a fast, accurate and cheap analytical technique. Moreover, it enables to measure simultaneous attributes with a simple sample preparation. Therefore, it could be a suitable alternative to chemical conventional methods. Previous studies have showed that NIRS technique is a good predictor of intramuscular fat (IMF) and protein content in meat with higher prediction accuracy for IMF (Prevolnik et al., 2004; Prieto et al., 2009). Fatty acid content has also been predicted 
by NIRS; nevertheless, this technique has a limited ability for estimating some individual FA due to the similarity between their NIR absorption patterns (Windham and Morrison, 1998). In rabbit, NIRS has been applied for predicting fat and protein content in meat (Masoero et al., 1994; Pla et al., 2004). To our knowledge, there are not studies about NIRS predictions of FA profile in IMF in rabbits, since Pla et al. (2007) studied FA in hind leg meat, including inter- and intramuscular fat.

Rabbit selection programmes focused on IMF are being conducted in our institute. These programmes need a substantial amount of data, thus fast and accurate methods are required. NIRS could be a suitable alternative to study IMF and possible changes in FA profile in all individuals involved in genetic programmes.

The objective of this study was to evaluate the potential use of NIRS for predicting FA and IMF content in the Longissimus muscle to be applied in rabbit selection programmes.

\section{MATERIALS AND METHODS}

\subsection{Animals and meat samples}

All experimental procedures involving animals were approved by the Research Ethics Committee of the Universitat Politècnica de València. A total of 143 rabbits (61 females and 82 males) were used in this experiment. To ensure enough variability in the samples analysed, rabbits were from three different synthetic lines and were slaughtered between 5 and 61 weeks of age.

Animals were reared at the experimental farm of the Universitat Politècnica de València. From weaning ( 4 weeks of age) to 9 weeks of age, rabbits were reared collectively and were fed ad libitum with a commercial diet formulated for growing rabbits $(15.7 \%$ crude protein, $16.4 \%$ crude fiber, $3.0 \%$ fat). During the subsequent experimental period, rabbits were housed in individual cages and received a restricted feed with a diet formulated for adults ( $17 \%$ crude protein, $15.8 \%$ crude fiber, $3.5 \%$ fat). The amount of feed was 135 grams/day and was distributed once daily.

Rabbits were slaughtered by electrical stunning and exsanguination at the abattoir on the farm. No fasting was applied. After the slaughter, the carcasses were stored at $4^{\circ} \mathrm{C}$ 
during 24 hours and then the Longissimus muscles (LM) were excised from the carcass. Meat obtained from LM was ground, freeze-dried, vacuum-packed and stored at $80^{\circ} \mathrm{C}$ until analyses.

\subsection{Intramuscular fat analysis}

Total lipids were determined by ether extraction (Soxtec 2055, Tecator, Höganäs, Sweden) with a previous acid hydrolysis (Soxcap 2022, Tecator, Höganäs, Sweden) (ISO-R-1443) in triplicate from freeze-dried LM in 143 samples. Lipid content was expressed as grams per 100 grams of fresh tissue, this value was obtained taking into account the dry matter content determined from the weight of minced LM before and after freeze-drying.

\subsection{Protein analysis}

Determination of protein content was based on Total Nitrogen content by Kjeldahl procedure (ISO-R-937) using a Kjeltec 2300 Analyzer (Tecator, Höganäs, Sweden). Protein content was quantified in triplicate from freeze-dried LM in 122 samples. Results were expressed as grams per 100 grams of fresh tissue, this value was obtained taking into account the dry matter content determined from the weight of minced LM before and after freeze-drying.

\subsection{Fatty acid analysis}

Fatty acid profile of freeze-dried LM was determined in 123 samples. Fatty acid methyl esters (Fame) were prepared as described by O'Fallon et al. (2007) and were analysed in a Focus Gas Chromatograph (Thermo, Milan, Italy) equipped with a split/splitless injector and a flame ionization detector. The separation of methyl esters was performed in a fused silica capillary column SPTM 2560 (Supelco, PA, USA) (100 m x $0.25 \mathrm{~mm} \times 0.2 \mu \mathrm{m}$ film thickness). The carrier gas was Helium at a linear velocity of 20 $\mathrm{cm} / \mathrm{sec}$. The samples were injected with a split ratio of $1 / 100$. The initial oven temperature was set at $140^{\circ} \mathrm{C}$ held for $5 \mathrm{~min}$ and increased to 240 at $4^{\circ} \mathrm{C} / \mathrm{min}$ and finally maintained at that temperature for $30 \mathrm{~min}$. Both detector and injector temperatures were set at $260^{\circ} \mathrm{C}$. The individual FA were identified by comparing their retention times with standards of Fame supplied by Supelco (PA, USA) and quantified by using C21:0 as internal standard. 
Fatty acids were expressed as milligrams per 100 grams of fresh tissue, this value was obtained taking into account the dry matter content determined from the weight of minced LM before and after freeze-drying.

\subsection{NIRS analysis}

\section{Spectral data collection}

Once freeze-dried muscle samples reached room temperature, they were scanned between 1100 and $2498 \mathrm{~nm}$ with a monochromator (model 5000, NIRSystem INC., Silver Spring, MD, USA) equipped with a transport module using ISI software, version 3.10 from Infrasoft International (Infrasoft International LLC, State College, PA, USA). Absorbance data were recorded at $2 \mathrm{~nm}$ and stored as log (1/reflectance). Sample measurements were taken in circular cups with quartz windows of $3.8 \mathrm{~cm}$ diameter. A sample cup was filled, placed in NIRS unit and two spectra, rotating 90 degrees the sample cup, were obtained. The sample cup was refilled with the same sample and the procedure was repeated in order to obtain four spectra of each sample. The similarity between the four reflectance spectra was studied by using Root Mean Squared (RMS) statistic. Then, the four spectra were averaged.

\section{Pre-treatment of spectral data}

All chemometric analyses were performed using WinISI-4 version 1.60 from Infrasoft International and Foss (Infrasoft International LLC, State College, PA, USA and FOSS, Höganäs, Sweden). Spectral anomalous were identified using the Mahalanobis distance to the center of the population $(\mathrm{GH})$. Samples with a $\mathrm{GH}$ value higher than 3 were considered spectral outliers (Shenk and Westerhaus, 1996) and were eliminated from the population. Spectral data pre-treatments such as Standard Normal Variate (SNV) and Detrending (DT) and first or second derivative mathematical treatments were applied.

\section{Calibration development}

Once spectral outliers were removed, calibrations were performed using the WinISI-4 software version 1.60 (Infrasoft International LLC, State College, PA, USA and FOSS, Höganäs, Sweden). Prediction equations were obtained using Modified Partial Least Squares (MPLS) as regression method (Shenk and Westerhaus, 1996) for IMF, protein, 
FA groups, FA ratios and individual FA. Cross-validation was performed in order to select the optimal number of factors and avoid overfitting. Concentration outliers were identified by using T-statistic, which indicates the difference between the reference and the predicted value. Samples with a T-value higher than 2.5 were considered as concentration outliers (Shenk and Westerhaus, 1996), and the reference chemical analysis of was repeated. Just enough passes were performed to detect outliers. Critical value for $\mathrm{GH}$ outliers was set at 10 in this step. The cross-validation operated with 5 groups. Regression equations were obtained using different combinations of scatter correction (no correction, SNV, SNV+DT) and mathematical treatments: $(1,4,4,1),(2,4,4,1),(1,5,5,1)$ and $(2,5,5,1)$, where the first digit is the order of the derivative, the second is the gap over which the derivative is calculated, the third is the number of data points used in the first smoothing, and the fourth is the number of data points used in the second smoothing. The best equation for each parameter was selected attending to Standard Error of Cross-Validation (SECV) and Determination Coefficient of Cross Validation $\left(R^{2} \mathrm{CV}\right)$. Moreover, the Residual Predictive Deviation $\left(R P D_{C V}\right)$, defined as the ratio between standard deviation of reference data to the SECV, was used to evaluate the predictive ability of the calibration models (Williams, 2001). This author suggested RPD values of 1.6 to 2.0 for very rough screening, 2.1 to 2.5 for rough screening, 2.6 to 3.0 for screening purposes and higher than 3.0 for suitable prediction models.

\subsection{Statistical analysis}

Descriptive statistics of reference chemical data for IMF, protein, FA profile (groups and individual) and FA ratios were computed by the SAS statistical package (SAS Institute Inc. Cary, USA, 2002).

\section{RESULTS AND DISCUSSION}

\subsection{Chemical data}

Descriptive statistics for IMF, protein, FA groups and FA ratios of LM used in the calibration are summarized in Table 1. LM showed a low fat (1.32 g/100g muscle) and high protein content $(22.5 \mathrm{~g} / 100 \mathrm{~g}$ muscle) since it is the leanest muscle of the carcass. Similar values for fat and protein content were found by Pla et al. (2004) in this muscle. 
Intramuscular fat showed a wide range of variability $(C V \times 100=40.2)$, which is essential to obtain successful prediction equations. As expected, protein content had a lower variation $(C V \times 100=4.8)$.

Table 1. Descriptive statistics for intramuscular fat (IMF) $(n=139)$, protein $(n=120)$, fatty acid groups and fatty acid ratios $(\mathrm{n}=119)$ in rabbit Longissimus muscle of the calibration set

\begin{tabular}{llllll}
\hline \hline Parameter & Mean & SD $^{1}$ & Min $^{2}$ & Max $^{3}$ & CVx100 $^{4}$ \\
\hline IMF (g/100 g muscle) & 1.32 & 0.53 & 0.75 & 3.25 & 40.2 \\
Protein (g/100 g muscle) & 22.5 & 1.1 & 18.1 & 26.3 & 4.8 \\
Groups (mg/100 g muscle) & & & & & \\
SFA $^{5}$ & 352 & 164 & 162 & 858 & 46.5 \\
MUFA $^{6}$ & 266 & 162 & 92 & 778 & 61.0 \\
PUFA $^{7}$ & 319 & 89 & 143 & 568 & 28.0 \\
n-6 PUFA & 264 & 80 & 110 & 493 & 30.2 \\
n-3 PUFA & & 11.4 & 23.6 & 82.2 & 21.1 \\
Ratios (mg/100 g muscle) & 54.3 & & & & \\
PUFA/SFA & 0.98 & 0.21 & 0.51 & 1.61 & 21.4 \\
n-6/n-3 & 4.87 & 0.87 & 2.94 & 7.27 & 17.9
\end{tabular}

${ }^{1} \mathrm{SD}$, standard deviation; ${ }^{2} \mathrm{Min}$, minimum; ${ }^{3} \mathrm{Max}$, maximum; ${ }^{4} \mathrm{CV}$, coefficient of variation; ${ }^{5} \mathrm{SFA}$, saturated fatty acids $=\mathrm{C} 14: 0+\mathrm{C} 15: 0+\mathrm{C} 16: 0+\mathrm{C} 17: 0+\mathrm{C} 18: 0 ;{ }^{6}$ MUFA, monounsaturated fatty acids $=C 16: 1+C 18: 1 n-9+C 18: 1 n-7 ; \quad{ }^{7}$ PUFA, polyunsaturated fatty acids $=C 18: 2 n-6+C 18: 3 n-$ $3+C 20: 2 n-6+C 20: 3 n-6+C 20: 4 n-6+C 20: 5 n-3+C 22: 4 n-6+C 22: 5 n-3+C 22: 6 n-3 ;{ }^{8} n-6$ PUFA $=C 18: 2 n-$ $6+C 20: 2 n-6+C 20: 3 n-6+C 20: 4 n-6+C 22: 4 n-6 ;{ }^{9} n-3$ PUFA $=C 18: 3 n-3+C 20: 5 n-3+C 22: 5 n-3+C 22: 6 n-3$.

The main FA in rabbit LM were saturated (SFA) and polyunsaturated (PUFA), with percentages around $38 \%$ and $34 \%$ of total FA, respectively. Monounsaturated (MUFA) FA represented lower percentage (28\%). Among PUFA, n-6 FA were the most abundant with percentages of $28 \%$, while n-3 FA were less represented (6\%). PUFA/SFA and $n$ $6 / n-3$ ratios, used to evaluate the nutritional quality of fat, showed values close to the nutritional recommendations (higher than 0.45 for PUFA/SFA and lower than 4 for $n$ 6/n-3) (reviewed by Hernández and Dalle Zotte, 2010). SFA and MUFA content had a high variability (CVx100 of 46.5 and 61.0 , respectively), but PUFA as well n-6, n-3 and FA ratios showed a lower variability (CVx100 between 17.9 and 30.2). 
Table 2. Descriptive statistics for individual fatty acids in rabbit Longissimus muscle of the calibration set $(n=119)$

\begin{tabular}{llllll}
\hline \hline Parameter (mg/100 g muscle) & Mean & SD $^{1}$ & Min $^{2}$ & Max $^{3}$ & CVx100 $^{4}$ \\
\hline C14:0 (myristic) & 18.3 & 13.4 & 3.71 & 62.5 & 73.5 \\
C15:0 (pentadecanoic) & 4.43 & 2.18 & 0.31 & 10.8 & 49.2 \\
C16:0 (palmitic) & 251 & 122 & 113 & 621 & 48.9 \\
C16:1 (palmitoleic) & 28.2 & 27.1 & 3.41 & 120 & 95.9 \\
C17:0 (heptadecanoic) & 6.18 & 2.70 & 2.08 & 15.0 & 43.7 \\
C18:0 (stearic) & 72.7 & 24.8 & 39.4 & 153 & 34.1 \\
C18:1 n-7 (vaccenic) & 13.8 & 6.8 & 3.79 & 38.1 & 49.4 \\
C18:1 n-9 (oleic) & 224 & 130 & 78.1 & 620 & 58.3 \\
C18:2 n-6 (linoleic) & 194 & 73.4 & 52.9 & 419 & 37.9 \\
C18:3 n-3 (linolenic) & 14.8 & 8.9 & 1.37 & 41.8 & 59.7 \\
C20:2 n-6 (eicosadienoic) & 2.33 & 0.80 & 0.45 & 4.80 & 34.2 \\
C20:3 n-6 (eicosatrienoic) & 4.05 & 0.81 & 2.21 & 6.47 & 20.0 \\
C20:4 n-6 (arachidonic) & 48.1 & 9.2 & 32.4 & 71.5 & 19.1 \\
C20:5 n-3 (eicosapentanoic) & 11.9 & 4.2 & 0.79 & 22.2 & 35.3 \\
C22:4 n-6 (docosatetraenoic) & 15.9 & 2.6 & 10.4 & 23.3 & 16.2 \\
C22:5 n-3 (docosapentanoic) & 7.11 & 1.86 & 4.39 & 12.3 & 26.1 \\
C22:6 n-3 (docosahexanoic) & 20.5 & 6.6 & 8.52 & 42.3 & 32.2 \\
\hline SD, standard deviation; ${ }^{2}$ (1) & & & & & \\
\hline
\end{tabular}

${ }^{1} \mathrm{SD}$, standard deviation; ${ }^{2} \mathrm{Min}$, minimum; ${ }^{3} \mathrm{Max}$, maximum; ${ }^{4} \mathrm{CV}$, coefficient of variation.

Table 2 shows descriptive statistics for individual FA of rabbits used in the calibration. The most ubiquitous FA in LM were palmitic (C16:0), oleic (C18:1 n-9) and linoleic (C18:2 n-6) acids, showing percentages of $27 \%, 24 \%$ and $21 \%$, respectively. Stearic (C18:0) and arachidonic acids (C20:4 $n-6)$ were also important with percentages around $8 \%$ and $5 \%$, repectively. Linolenic acid (C18:3n-3) and some long chain PUFA (i.e. C20:5 n-3, C22:4 n-6 and C22:6n-3) were also present in rabbit meat although at a lower content. The FA composition of rabbit LM was in agreement with that observed in previous studies (reviewed by Hernández and Gondret, 2006). Most individual FA showed a wide range of variation, mainly C14:0, C16:1, C18:1 n-9 and C18:3 n-3 (CVx100 between 58.3 and 95.9). The variability among calibration set found in this study was in similar range as in other works in beef (Sierra et al., 2008) and lamb (Guy et al., 2011) meat for the most of parameters. 


\subsection{NIRS calibrations}

Calibration statistics for IMF, protein, FA groups and FA ratios are reported in Table 3. The parameters corresponding to IMF calibration indicated good prediction ability $\left(R^{2} c V=0.98\right.$ and $\left.R P D_{C V}=7.57\right)$. Accurate NIRS calibrations for IMF have also been reported in poultry, beef and pork meat (reviewed by Prieto et al., 2009). In rabbit meat, Masoero et al. (1994) and Pla et al. (2004) also obtained good prediction equations for fat content which included inter- and intramuscular fat. The calibration model for protein content had a low $R^{2} c v(0.77)$ and $R P D_{c v}(2.07)$, but it could be adequate for rough screening (Williams, 2001). A lower accuracy of protein prediction had been previously observed in beef, pork and poultry meat (reviewed by Prieto et al., 2009). These authors proposed as possible causes a narrow range on variability within the calibration set and analytical differences between Kjeldahl and NIRS methodology, which is in accordance with our results (CVx100 of protein=4.8).

Equations for SFA and MUFA content (Table 3 ) showed good accuracy $\left(R^{2} c v\right.$ of 0.96 and 0.98 and $R^{2} D_{C V}$ of 5.09 and 6.69, respectively). Prediction models for PUFA and n-6 FA content were less accurate $\left(R^{2} c v=0.83\right.$ and $0.87, R P D_{C V}=2.40$ and 2.82 , respectively), indicating suitable predictions for screening. Results for n-3 FA content indicated no accurate predictions $\left(\mathrm{R}^{2} \mathrm{CV}=0.50\right.$ and $\left.R P D_{C V}=1.41\right)$. The higher accuracies for SFA and MUFA compared to PUFA content found in this study are in line with findings of other authors (Sierra et al., 2008; Guy et al., 2011), and might be related to the narrow range of variability in PUFA content (Table 1) and a less ability of NIRS to detect the higher double bonds presents in PUFA. Regarding $n-6$ and n-3 FA content predictions, a similar pattern was observed by Pla et al. (2007); the lower accuracy for n-3 FA prediction might be due to a lower presence and variability of n-3 FA in rabbit meat. Equations for FA ratios had low accuracies $\left(R^{2} c V=0.81\right.$ for PUFA/SFA ratio and $R^{2} c V=0.64$ for $n-6 / n-3$ ratio), only suitable for rough screening. No information was found about prediction of ratios in the literature using NIRS. Nevertheless, FA ratios predictions could be interesting from the point of view of nutritional quality of meat. NIRS technology provides a direct prediction of the ratio with its prediction error. This is more suitable than obtaining two parameters separately and calculating its ratio, since its standard error cannot be estimated directly. 
Table 3. Statistical parameters of equations for near infrared reflectance spectroscopy calibrations of intramuscular fat (IMF), protein, fatty acid content and fatty acid ratios in rabbit Longissimus muscle

\begin{tabular}{|c|c|c|c|c|c|c|c|}
\hline Parameter & $N^{1}$ & Mean & SD $^{2}$ & Interval & $\mathbf{R}^{2} \mathrm{cv}^{3}$ & SECV $^{4}$ & $\mathrm{RPD}_{\mathrm{cv}}{ }^{5}$ \\
\hline IMF (g/100 g muscle) & 137 & 1.32 & 0.53 & $0.75-3.25$ & 0.98 & 0.07 & 7.57 \\
\hline Protein (g/100 g muscle) & 106 & 22.5 & 0.85 & $20.4-24.3$ & 0.77 & 0.41 & 2.07 \\
\hline \multicolumn{8}{|l|}{ Groups (mg/100 g muscle) } \\
\hline SFA $^{6}$ & 119 & 352 & 164 & $162-858$ & 0.96 & 32.2 & 5.08 \\
\hline MUFA $^{7}$ & 116 & 263 & 162 & $91.7-778$ & 0.98 & 24.2 & 6.68 \\
\hline PUFA $^{8}$ & 119 & 319 & 89 & $143-568$ & 0.83 & 37.2 & 2.40 \\
\hline n-6 PUFA ${ }^{9}$ & 117 & 262 & 78 & $110-493$ & 0.87 & 27.8 & 2.82 \\
\hline n-3 PUFA ${ }^{10}$ & 117 & 54.4 & 11.1 & $31.3-82.2$ & 0.50 & 7.87 & 1.40 \\
\hline \multicolumn{8}{|l|}{ Ratios (mg/100 g muscle) } \\
\hline PUFA/SFA & 116 & 0.97 & 0.20 & $0.51-1.44$ & 0.81 & 0.09 & 2.25 \\
\hline$n-6 / n-3$ & 116 & 4.84 & 0.82 & $3.15-6.59$ & 0.64 & 0.49 & 1.66 \\
\hline
\end{tabular}

${ }^{1} \mathrm{~N}$, number of samples, ${ }^{2} \mathrm{SD}$, standard deviation, ${ }^{3} \mathrm{R}^{2} \mathrm{cv}$, coefficient of determination of crossvalidation, ${ }^{4} \mathrm{SECV}$, standard error of cross validation, ${ }^{5} \mathrm{RPD} \mathrm{DV}_{\mathrm{CV}}, \mathrm{SD} / \mathrm{SECV}$. ${ }^{6} \mathrm{SFA}$, saturated fatty acids $=C 14: 0+C 15: 0+C 16: 0+C 17: 0+C 18: 0 ;{ }^{7}$ MUFA, monounsaturated fatty acids $=C 16: 1+C 18: 1 \mathrm{n}-$ 9+C18:1n-7; ${ }^{8}$ PUFA, polyunsaturated fatty acids $=C 18: 2 n-6+C 18: 3 n-3+C 20: 2 n-6+C 20: 3 n-$ $6+C 20: 4 n-6+C 20: 5 n-3+C 22: 4 n-6+C 22: 5 n-3+C 22: 6 n-3 ; \quad{ }^{9} n-6=\quad C 18: 2 n-6+C 20: 2 n-6+C 20: 3 n-$ $6+C 20: 4 n-6+C 22: 4 n-6 ;{ }^{10} n-3=C 18: 3 n-3+C 20: 5 n-3+C 22: 5 n-3+C 22: 6 n-3$.

Calibration equation results for individual FA content are shown in Table 4. The best calibration equations were found for $\mathrm{C} 18: 1 \mathrm{n}-9, \mathrm{C} 16: 0$ and $\mathrm{C} 18: 3 \mathrm{n}-3$ with $\mathrm{R}^{2} \mathrm{cv}$ higher than 0.95. Accurate equations were also obtained for C14:0, C15:0, C16:1, C17:0, C18:0 and C18:2 n-6 with $R^{2}$ cv between 0.91 and 0.94. RPD ${ }_{c v}$ statistics of these equations showed values higher than those recommended in literature for suitable prediction models (Williams, 2001). Other minor FA as C18:1 n-7 presented less accurate predictions $\left(\mathrm{R}^{2} \mathrm{CV}=0.82\right.$ and $\left.\mathrm{RPD} \mathrm{CV}_{\mathrm{C}}=2.37\right)$, only adequate for rough screening. Inferior results were obtained for long chain PUFA such as C20:2 n-6, C20:4 n-6, C20:5 n-3 and $C 22: 5 n-3\left(R^{2} c v\right.$ between 0.61 and 0.73 and $R P D_{C V}$ between 1.60 and 1.95) indicating proper models for very rough screening. Finally, C20:3 n-6, C22:4 n-6 and C22:6 n-3 models were unacceptable for predictions $\left(R^{2} c v\right.$ between 0.12 and 0.57 and $R P D_{c v}$ between 1.06 and 1.52). 
Table 4. Statistical parameters of equations for near infrared reflectance spectroscopy calibrations of individual fatty acid content in rabbit Longissimus muscle

\begin{tabular}{|c|c|c|c|c|c|c|c|}
\hline $\begin{array}{l}\text { Parameter } \\
\text { ( } \mathrm{mg} / 100 \mathrm{~g} \text { muscle) }\end{array}$ & $\mathbf{N}^{1}$ & Mean & $S^{2}$ & Interval & $\mathbf{R}^{2} \mathrm{cv}^{3}$ & SECV $^{4}$ & $\mathrm{RPD}_{\mathrm{cv}}{ }^{5}$ \\
\hline C14:0 & 116 & 17.5 & 12.5 & $3.71-59.0$ & 0.94 & 2.96 & 4.23 \\
\hline C15:0 & 116 & 4.42 & 2.14 & $1.25-10.8$ & 0.91 & 0.63 & 3.38 \\
\hline C16:0 & 118 & 249 & 121 & $113-621$ & 0.96 & 24.5 & 4.93 \\
\hline C16:1 & 115 & 27.1 & 26.6 & $3.41-120$ & 0.92 & 7.42 & 3.59 \\
\hline C17:0 & 113 & 5.91 & 2.43 & $2.08-13.6$ & 0.91 & 0.75 & 3.24 \\
\hline C18:0 & 116 & 71.3 & 23.4 & $39.4-153$ & 0.90 & 7.33 & 3.20 \\
\hline C18:1 n-7 & 117 & 13.8 & 6.9 & $3.79-38.1$ & 0.82 & 2.90 & 2.37 \\
\hline C18:1 n-9 & 116 & 221 & 130 & $78.1-620$ & 0.97 & 21.3 & 6.10 \\
\hline C18:2 n-6 & 115 & 190 & 71 & $52.9-419$ & 0.91 & 21.3 & 3.33 \\
\hline C18:3 n-3 & 113 & 14.3 & 8.6 & $1.37-41.8$ & 0.94 & 2.18 & 3.93 \\
\hline$C 20: 2$ n-6 & 114 & 2.30 & 0.75 & $0.58-4.80$ & 0.72 & 0.39 & 1.92 \\
\hline$C 20: 3 n-6$ & 115 & 3.99 & 0.76 & $2.21-5.87$ & 0.57 & 0.50 & 1.52 \\
\hline$C 20: 4 n-6$ & 117 & 47.8 & 9.0 & $32.4-68.5$ & 0.61 & 5.60 & 1.60 \\
\hline C20:5 n-3 & 115 & 12.0 & 3.9 & $3.61-20.5$ & 0.73 & 2.01 & 1.95 \\
\hline C22:4 n-6 & 118 & 15.8 & 2.5 & $10.4-21.9$ & 0.12 & 2.34 & 1.06 \\
\hline$C 22: 5 n-3$ & 115 & 7.04 & 1.84 & $4.39-12.3$ & 0.73 & 0.95 & 1.94 \\
\hline C22:6 n-3 & 117 & 20.2 & 6.25 & $8.52-36.5$ & 0.38 & 4.95 & 1.26 \\
\hline
\end{tabular}

${ }^{1} \mathrm{~N}$, number of samples, ${ }^{2} \mathrm{SD}$, standard deviation, ${ }^{3} \mathrm{R}^{2} \mathrm{cv}$, coefficient of determination of crossvalidation, ${ }^{4} \mathrm{SECV}$, standard error of cross validation, ${ }^{5} \mathrm{RPD} \mathrm{cv}, \mathrm{SD} / \mathrm{SECV}$.

Comparisons between studies are difficult due to the use of different equipment, wavelength range, sample preparation and chemical analyses. Fatty acid data were commonly expressed as percentage of the total FA when used for prediction by NIRS in previous studies in rabbit (Pla et al., 2007), beef (Windham and Morrison, 1998) and pork meat (González-Martín et al., 2005). However, more recently studies in beef (Sierra et al., 2008; Prieto et al., 2011) and lamb meat (Guy et al., 2011) used reference data expressed as concentration ( $\mathrm{mg}$ or $\mathrm{g}$ ) in the muscle and obtained higher accuracies of prediction. This work is the first analysis of FA content of IMF by NIRS in rabbit and FA content was expressed as concentration (mg) in the muscle. Calibrations for the main FA content in rabbit LM were similar to those obtained by Guy et al. 
(2011) in ground lamb meat. These authors found accurate prediction models for C16:0, C18:0, C18:1 n-9 FA; however, we obtained higher accuracies for C18:2 n-6 and C18:3 n-3 predictions. Higher errors for the prediction of several long chain PUFA, present at al lower amount in rabbit meat, were also found in other works (Sierra et al., 2008; Guy et al., 2011). The prediction accuracies obtained in this study were higher than those obtained by Pla et al. (2007) in hind leg meat for most individual FA except for C18:2 n-6, C20:4 n-6 and 20:3 n-6, which were similar.

\section{CONCLUSIONS}

Accurate predictions were obtained for IMF, SFA and MUFA content as well as for the main individual FA content in rabbit meat (C16:0, C18:1 n-9 and C18:2 n-6) and other FA found in lower amounts (C14:0, C15:0, C16:1, C17:0, C18:0 and C18:3 n-3). Less accurate predictions were obtained for protein content, PUFA, n-6, n-3, FA ratios as well as C18:1 n-7 and long chain PUFA. NIRS can be a suitable alternative to chemical conventional methods to predict IMF and its FA content in rabbit meat for being used in genetic programmes. The models developed in this study will be applied in rabbit selection programmes of our institute.

\section{LITERATURE CITED}

González-Martín, I., C. González-Pérez, N. Álvarez-García, and J. M. González-Cabrera. 2005. On-line determination of fatty acid composition in intramuscular fat of Iberian pork by NIRS with a remote reflectance fibre optic probe. Meat Science. 69:243-248.

Guy, F., A. Prache, D. Thomas, D. Bauchart, and D. Andueza. 2011. Prediction of lamb meat fatty acid composition using near-infrared reflectance spectroscopy (NIRS). Food Chemistry. 127:1280-1286.

Hernández, P., and F. Gondret. 2006. Rabbit meat quality and safety. In: L. Maertens and P. Coudert, editors, Recent Advances in Rabbit Sciences. ILVO, Melle, Belgium. p. $267-290$

Hernández, P., and A. Dalle Zotte. 2010. Influence of diet on rabbit meat quality. In: C. de Blas, and J. Wiseman, editors, Nutrition of the rabbit. CAB International. Wallinford, Oxon, UK. p. 163-178. 
O’Fallon, J. V., J. R. Busboom, M. L. Nelson, and C. T. Gaskins. 2007. A direct method for fatty acid methyl ester synthesis: Application to wet meat tissue, oils, and feedstuffs. Journal of Animal Science. 85:1511-1521.

Masoero, G., G. Xiccato, A. Dalle Zotte, R. Parigi-Bini, and G. Bergoglio. 1994. Analysis of freeze-dried rabbit meat by NIRS. Zootecnia Nutrizione Animale. 20:319-329.

Pla, M., M. Pascual, and B. Ariño. 2004. Protein, fat and moisture content of retail cuts of rabbit meat evaluated with the NIRS methodology. World Rabbit Science. 12:149-158.

Pla, M., P. Hernández, B. Ariño, J. A. Ramírez, and I. Díaz. 2007. Prediction of fatty acid content in rabbit meat and discrimination between conventional and organic production systems by NIRS methodology. Food Chemistry. 100:165-170.

Prevolnik, M., M. Candek-Potokar, and D. Skorjanc. 2004. Ability of NIR spectroscopy to predict meat chemical composition and quality- a review. Czech Journal of Animal Science. 49: 500-510.

Prieto, N., R. Roehe, P. Lavín, G. Batten, and S. Andrés. 2009. Application of near infrared reflectance spectroscopy to predict meat and meat products quality: A review. Meat Science. 83:175-186.

Prieto, N., D. W. Ross, E. A.Navajas, R. I. Richardson, J. J. Hyslop, G. Simm, and R. Roehe. 2011. Online prediction of fatty acid profile in crossbred Limousin and Aberdeen Angus beef cattle using near infrared reflectance spectroscopy. Animal. 5:155-16.

SAS 2002. SAS/STAT User's guide (Release 9.1) Statistical Analysis System Institute Inc, Cary, NC, USA.

Shenk, J. S., and M. O. Westerhaus. 1996. Calibration the ISI way. In: A.M.C. Davies, and P. Williams, editors, Near Infrared Spectroscopy: The Future Waves. NIR Publications. Chichester, UK. p. 198-202.

Sierra, V., N. Aldai, P. Castro, K. Osoro, A. Coto-Montes, and M. Olivan. 2008. Prediction of the fatty acid composition of beef by near infrared transmittance spectroscopy. Meat Science. 78:248-255.

Williams, P.C. 2001. Implementation of Near-Infrared Technology. In P. C. Williams and K. Norris, editors, Near-Infrared Technology in the Agricultural and Food Industries. American Association of Cereal Chemists. St. Paul, Minnesota, USA. p.145-169. 
Windham, W. R., and W. H. Morrison. 1998. Prediction of fatty acid content in beef neck lean by near infrared reflectance analysis. Journal of Near Infrared Spectroscopy. 6:229-234. 